University of Nebraska - Lincoln

DigitalCommons@University of Nebraska - Lincoln

3-7-2008

\title{
Piezoresponse Force Microscopy Studies of Switching Behavior of Ferroelectric Capacitors on a 100-ns Time Scale
}

\author{
Alexei Gruverman \\ University of Nebraska-Lincoln, agruverman2@unl.edu \\ D. $\mathrm{Wu}$ \\ North Carolina State University \\ J. F. Scott \\ Cambridge University
}

Follow this and additional works at: https://digitalcommons.unl.edu/physicsgruverman

Part of the Physics Commons

Gruverman, Alexei; Wu, D.; and Scott, J. F., "Piezoresponse Force Microscopy Studies of Switching Behavior of Ferroelectric Capacitors on a 100-ns Time Scale" (2008). Alexei Gruverman Publications. 1. https://digitalcommons.unl.edu/physicsgruverman/1

This Article is brought to you for free and open access by the Research Papers in Physics and Astronomy at DigitalCommons@University of Nebraska - Lincoln. It has been accepted for inclusion in Alexei Gruverman Publications by an authorized administrator of DigitalCommons@University of Nebraska - Lincoln. 


\title{
Piezoresponse Force Microscopy Studies of Switching Behavior of Ferroelectric Capacitors on a 100-ns Time Scale
}

\author{
A. Gruverman, ${ }^{1, *}$ D. Wu, ${ }^{2}$ and J. F. Scott ${ }^{3}$ \\ ${ }^{1}$ Department of Physics and Astronomy, University of Nebraska-Lincoln, Lincoln, Nebraska, USA \\ ${ }^{2}$ Department of Physics, North Carolina State University, Raleigh, North Carolina, USA \\ ${ }^{3}$ Earth Sciences Department, Cambridge University, Cambridge CB2 3EQ, United Kingdom
}

(Received 12 September 2007; published 5 March 2008)

Piezoresponse force microscopy is a powerful technique for nm-scale studies but is usually limited by response time. In this Letter, we report the first direct studies of ferroelectric capacitor switching on a submicrosecond time scale. Simultaneous domain imaging and sub- $\mu$ s transient current measurements establish a direct relationship between polarization $P(t)$ and domain kinetics. Switching times scale with capacitor size over an order of magnitude. Small capacitors, where polarization reversal is dominated by domain wall motion, switch faster at high fields but more slowly at low fields while larger capacitors do the reverse.

PACS numbers: 77.80.Fm, 68.37.Yz, 77.84.Dy

Among nonvolatile memory technologies, ferroelectric random access memory (FeRAM) is one of the most promising and technologically advanced [1]. Manufacturers of FeRAMs have recently lowered the film thickness below $100 \mathrm{~nm}$, which introduces thickness scaling of both static properties and dynamic switching parameters of ferroelectric capacitors. Similarly, the domain geometry and switching times depend also upon capacitor lateral dimensions. However, there are no theories to predict how the switching times depend upon domain kinetics, and reports conflict on whether submicron devices switch more slowly or less slowly than larger ones, leaving open one of the most important questions in the development of ferroelectric-based nanotechnologies [2,3].

The classical nucleation theory of Kolmogorov, Avrami, and Ishibashi (KAI) [4-6] considers the switching process as the phase transformation in infinite media with a constant nucleation rate, and with domain wall velocity as a rate-limiting parameter. However, this is algebraically incompatible with finite-size structures [7]. In the case of polycrystalline ferroelectric films, nucleation-limited models (NLS) [8,9] provide a better description of the switching kinetics. Qualitatively different switching behavior has been reported for submicron capacitors versus larger ones, presumably due to the strong effect of the electrode perimeter resulting in larger contribution of wall motion to polarization reversal $[10,11]$. Clearly, to determine how the intrinsic switching behavior changes as a function of the capacitor size and to achieve better theoretical interpretation of the experimental data, direct studies of the domain switching kinetics in confined ferroelectric structures are necessary. A major limitation in acquiring this crucial information is the lack of experimental methods to characterize the domain kinetics in ferroelectric thin films $[12,13]$. The lateral dimensions of the ferroelectric capacitors in FeRAM devices are in the micrometer range with the switching times measuring well below $100 \mathrm{~ns}[14,15]$, which requires testing of domain switching behavior with the nanometer spatial and nanosecond time resolutiondone for the first time in this Letter. In the absence of such local data, only rudimentary attempts have been made to develop microscopic switching models in finite-size structures $[16,17]$.

We show that very small capacitors switch more rapidly at the high-field range, but more slowly at low fields due to different activation energies in the two cases and an exponential field dependence of switching times. Visualization of instantaneous domain configurations by means of piezoresponse force microscopy (PFM) $[18,19]$ in conjunction with transient current measurements allows us to establish a direct relationship between the electrically measured polarization reversal signal and domain kinetics and determine the relative contribution of nucleation and wall motion mechanisms into polarization reversal.

We investigated the switching behavior of 180 -nm thick tetragonal $\mathrm{Pb}(\mathrm{Zr}, \mathrm{Ti}) \mathrm{O}_{3}$ (PZT) capacitors with a Pt bottom electrode. Reactive ion etching has been used to fabricate 50 -nm thick sputter-deposited $\mathrm{IrO}_{2}$ top electrodes with $1 \times$ $1.5 \mu \mathrm{m}^{2}, 3 \times 3 \mu \mathrm{m}^{2}$ and $5 \times 5 \mu \mathrm{m}^{2}$ lateral dimensions (area ratios of 16:1).

In PFM imaging of capacitors, an external ac bias applied to the top electrode generates a uniform electrical field within the capacitor so that the PFM tip senses the local piezoelectric response from the whole thickness of the ferroelectric layer. By scanning the top electrode with the voltage-modulated tip, a topographic image of the capacitor as well as two-dimensional maps of piezoresponse amplitude and phase signals can be generated providing information on polarization distribution in the capacitor at the nanoscale [Figs. 1(a)-1(c)]. An imaging ac voltage of $10 \mathrm{kHz}, 0.3 \mathrm{~V}$ rms has been used for domain imaging. An additional $0.1 \mathrm{~V} \mathrm{dc}$ offset was applied to the tip during PFM imaging to account for an internal bias in the capacitors and to stabilize the domain patterns. 

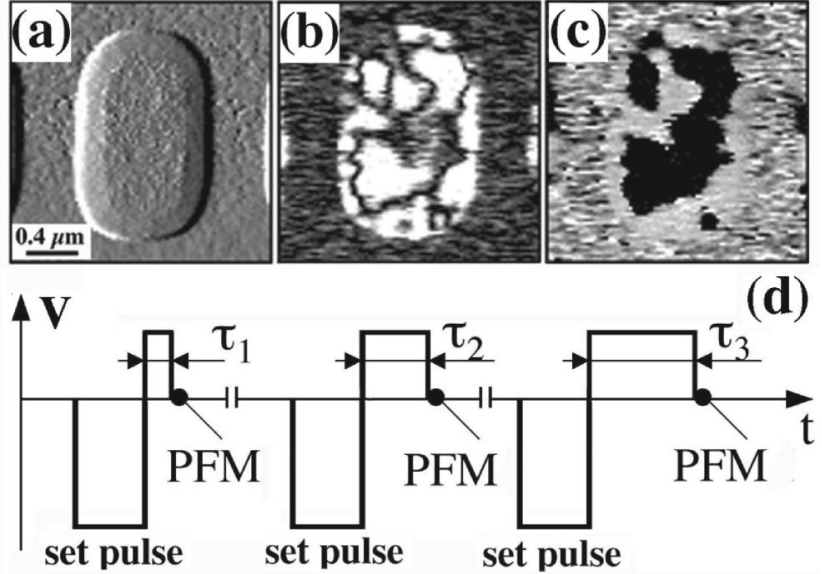

FIG. 1. (a) Topographic image of the $1 \times 1.5 \mu \mathrm{m}^{2}$ capacitor; (b) PFM amplitude and (c) PFM phase images of the initial domain structure in the same capacitor, (d) Voltage pulse sequence used to study domain kinetics in thin film capacitors using step-by-step switching. While amplitude of all the input voltage pulses is kept constant, pulse duration is incrementally increasing from cycle to cycle.

A commercial atomic force microscope (PSI Autoprobe M5), equipped with a pulse generator (Agilent 81101A) and evaluation module board (Texas Instruments THS5138) were used, with a 5-ns rise (18 ns when connected to the $5 \times 5 \mu \mathrm{m}^{2}$ capacitors). The switching currents have been measured using a standard pulse sequence called PUND (positive up negative down) [20]. Figures 2(a) and 2(b) show the switching transients for the $1 \times$ $1.5 \mu \mathrm{m}^{2}$ and $5 \times 5 \mu \mathrm{m}^{2}$ capacitors. Comparison of the switching times [Fig. 2(c)] shows that smaller capacitors switch faster than the larger ones in agreement with pre-
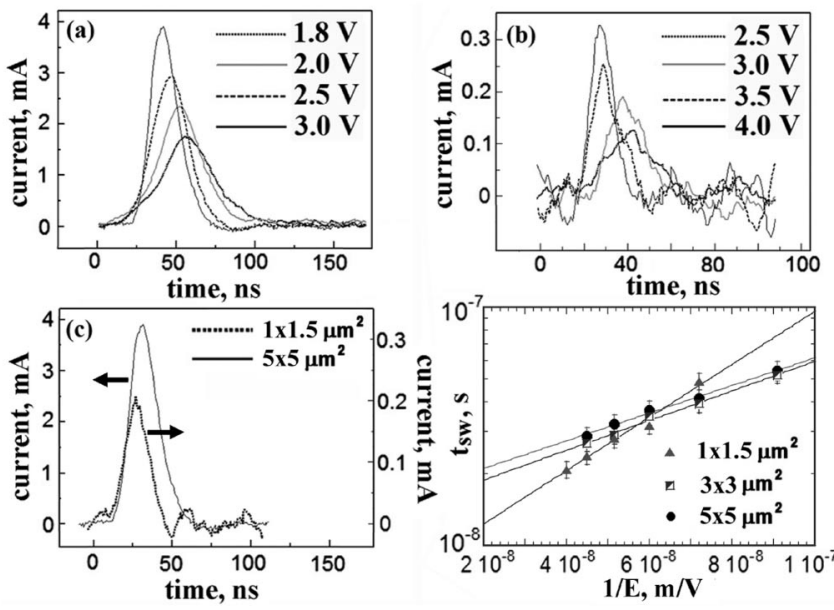

FIG. 2. Switching currents for different applied biases in $5 \times$ $5 \mu \mathrm{m}^{2}$ (a) and $1 \times 1.5 \mu \mathrm{m}^{2}$ (b) capacitors; (c) Comparison of the switching currents for different capacitors under the applied voltage of $3.0 \mathrm{~V}(E=167 \mathrm{kV} / \mathrm{cm}))$; (d) Field dependencies of switching times for different capacitors obtained from switching current measurements. viously reported data [21]. However, this relation is observed only in the high-field range. From the field dependencies of the switching times obtained from switching current measurements [Fig. 2(d)] it can be seen that in the low-field range (below $200 \mathrm{kV} / \mathrm{cm}$ ) the smaller capacitors actually switch more slowly [22]. This effect is likely due to a significant difference in the switching mechanisms as well in the activation fields measured in smaller and larger capacitors $\left(260 \mathrm{kV} / \mathrm{cm}\right.$ for $1 \times 1.5 \mu \mathrm{m}^{2}$ capacitors and approximately $140 \mathrm{kV} / \mathrm{cm}$ for larger capacitors). Previously, the absence of any significant capacitor size effect on activation field for capacitors larger than $10 \mu \mathrm{m}^{2}$ was reported [2]. However, our data indicate that the scaling effect onset occurs at a much smaller capacitor sizeseveral square microns and less - in agreement with estimations made by Scott et al. [23]. However, the main challenge still remains: direct assessment of the mechanism of scaling effect and relative contribution of nucleation and domain wall motion into polarization reversal. PFM visualization of domain kinetics has finally made this possible.

We investigated the domain switching kinetics by using a step-by-step switching approach in conjunction with PFM imaging [18]. The voltage pulse sequence used in our studies is shown in Fig. 1(d). In this approach an input voltage pulse shorter than the total switching time for a given pulse amplitude is applied to a capacitor generating only partial polarization reversal. An instantaneous domain configuration representing a certain stage of polarization reversal (corresponding to the pulse length) is registered by PFM. Domain switching kinetics is visualized by applying a series of input pulses with incrementally increasing duration and subsequent PFM imaging of domain structure in the capacitor after each pulse. At the beginning of each switching cycle, the capacitor is set into the opposite polarization state by applying a set pulse $(-5 \mathrm{~V}, 1 \mathrm{~s})$. This approach allows circumventing a problem of low time resolution in PFM and visualization of domain structure kinetics during submicrosecond switching process.

Figure 3 shows PFM images of instantaneous domain configurations developing in $1 \times 1.5 \mu \mathrm{m}^{2}, 3 \times 3 \mu \mathrm{m}^{2}$ and $5 \times 5 \mu \mathrm{m}^{2}$ capacitors at different stages of polarization reversal. Location of nucleation sites, nucleation density and rate, as well as lateral domain wall velocity can be directly measured from these data. It is worth emphasizing that in each switching cycle domain nucleation occurs in the same sites determined by the defect structure of the film-electrode interface, thus illustrating a role of inhomogeneous nucleation in polarization reversal. Here, we use a term "nucleation" to describe the emergence of new domains with polarization parallel to the applied field manifested by the inversed contrast in the PFM phase images.

PFM switching data in Fig. 3 indicates the difference in switching mechanisms in larger and smaller capacitors. In larger capacitors, two distinct stages of polarization reversal can be observed: a fast switching stage $(<1 \mu \mathrm{s})$ dominated by domain nucleation and a slower stage $(>1 \mu \mathrm{s})$ 

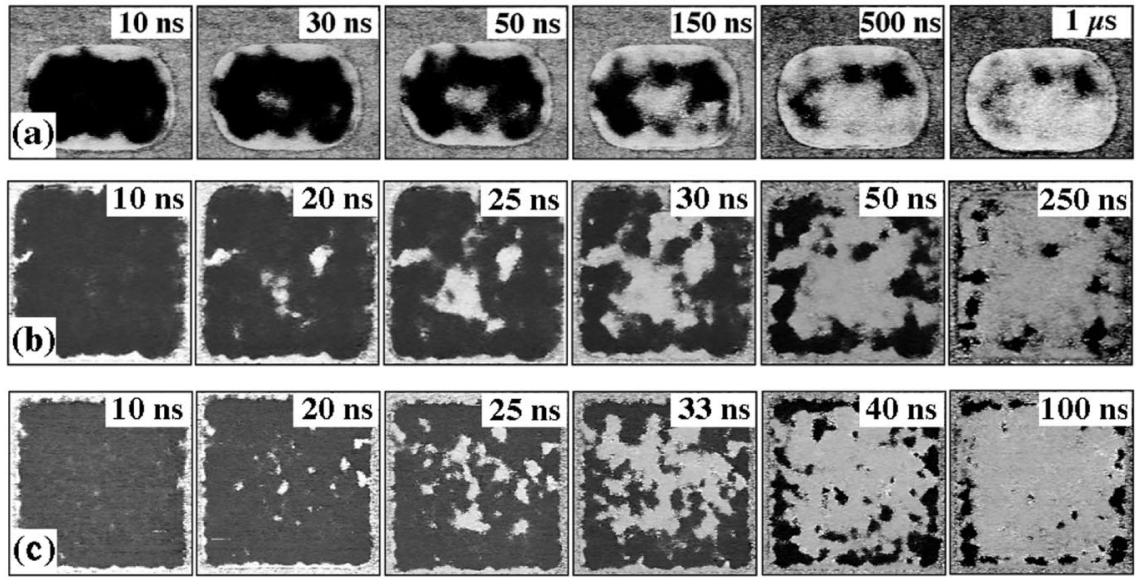

FIG. 3. PFM phase images of instantaneous domain configurations developing in (a) $1 \times 1.5 \mu \mathrm{m}^{2}$, (b) $3 \times 3 \mu \mathrm{m}^{2}$ and (c) $5 \times 5 \mu \mathrm{m}^{2}$ capacitors at different stages of polarization reversal process under the switching voltage of $1.8 \mathrm{~V}$ $(E=100 \mathrm{kV} / \mathrm{cm})$. The scanning area is (a) $1.4 \times 1.8 \mu \mathrm{m}^{2}, \quad$ (b) $3.2 \times$ $3.2 \mu \mathrm{m}^{2}$, and (c) $5.5 \times 5.5 \mu \mathrm{m}^{2}$. where switching occurs mainly via lateral domain wall motion. Approximately 80 domains nucleate in $5 \times 5 \mu \mathrm{m}^{2}$ capacitors during the 100-ns switching pulse, corresponding to the nucleation density of $3.2 \times 10^{12} \mathrm{~m}^{-2}$. In the $1 \times$ $1.5 \mu \mathrm{m}^{2}$ capacitors the switching proceeds mostly via lateral growth of just a few domains; i.e., the contribution of domain nucleation to polarization reversal is significantly reduced.

For quantitative analysis of domain kinetics, it is important to establish a relationship between switching current data and switched volume measurements by PFM. Figure 4(a) shows that the numerical integration of the switching current in Fig. 2(a) correlates well with the time dependence of the switched capacitor volume determined from PFM data in Fig. 3(c). It can be also seen that in the low-field range both $3 \times 3 \mu \mathrm{m}^{2}$ and $5 \times 5 \mu \mathrm{m}^{2}$ capacitors switch faster than the $1 \times 1.5 \mu \mathrm{m}^{2}$ capacitors, which is consistent with the switching current data in Fig. 2(d). Thus, PFM visualization of instantaneous domain configurations in conjunction with transient current measurements allows us to establish direct relationship between the electrically measured polarization reversal signal and domain switching kinetics.

To obtain further insight into the capacitor scaling effect on switching behavior we measured the field dependencies of lateral wall velocity and nucleation rate. The wall velocity has been measured by analyzing the time dependence of the radii of the growing domains visualized in the PFM snapshots (Fig. 3). This value has been averaged for several domains over the radius range of approximately $250 \mathrm{~nm}$.

It has been found that the nucleation rate does not stay constant throughout the switching but after reaching a maximum decreases to zero which corresponds to the complete transition from nucleation-dominated to wall motiondominated switching mechanism. A peak nucleation rate of $2.4 \times 10^{20} \mathrm{~s}^{-1} \mathrm{~m}^{-2}$ (for $E=100 \mathrm{kV} / \mathrm{cm}$ ) is 2 orders of magnitude lower than that estimated by Duiker et al. due to the lower defect concentration of PZT films we used.

The field dependence of the peak nucleation rate in the $5 \times 5 \mu \mathrm{m}^{2}$ capacitors is compared with the field dependence of the wall velocity in Fig. 4(b). Both nu- cleation rate $R$ and wall velocity $v$ are exponential functions of the applied field: $R=R_{\infty} \exp \left(-\alpha_{n} / E\right)$ and $v=$ $v_{\infty} \exp \left(-\alpha_{w} / E\right)$, where $\alpha_{n}=7.1 \times 10^{7} \mathrm{~V} / \mathrm{m}$ and $\alpha_{w}=$ $8.3 \times 10^{7} \mathrm{~V} / \mathrm{m}$. A difference between activation fields $\alpha_{n}$ and $\alpha_{w}$ explains faster switching of $1 \times 1.5 \mu \mathrm{m}^{2}$ capacitors in the high-field regime and allows estimation of the critical capacitor size for transition from wall limited to nucleation-limited switching.

The capacitor size criterion for the switching to be limited by the domain wall speed rather than by nucleation has been formulated by Scott under the assumption of the field-independent $v / R$ ratio [1,23]. The wall-speed limited switching time is determined by $t_{w}=\left(2 N R v^{2} / 9\right)^{-1 / 3}$, where $N$ is a density of nucleation sites. The nucleation time is given by $t_{n}=1 /(N R A)$, where $A$ is the capacitor lateral area. Hence, for nucleation time to have a negligible effect on switching it is required that $v /(N R) \ll A^{3 / 2}$, and for PZT capacitors yields that the critical size $A$ is $1 \mu \mathrm{m}^{2}$ [1]. A difference between $\alpha_{n}$ and $\alpha_{w}$ makes this criterion a function of the applied field:

$$
\frac{v_{\infty}}{N R_{\infty}} \exp \left(\frac{\alpha_{n}-\alpha_{v}}{E}\right) \ll A^{3 / 2} .
$$

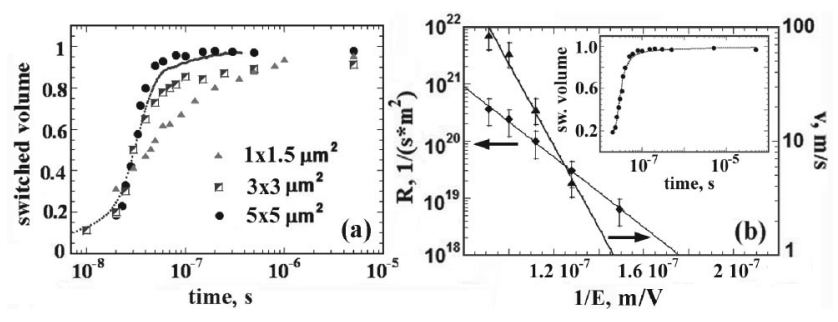

FIG. 4. (a) Switched capacitor volume as a function of time for different capacitors for the applied voltage of $1.8 \mathrm{~V}(E=$ $100 \mathrm{kV} / \mathrm{cm}$ ) measured by PFM showing faster switching in larger capacitors. Smaller capacitors start to switch faster in the fields above $200 \mathrm{kV} / \mathrm{cm}$. Dotted line-numerical integration of transient current in Fig. 2(a); (b) Nucleation rate and domain wall velocity vs electrical field for $5 \times 5 \mu \mathrm{m}^{2}$ capacitors. Inset: fitting of the PFM switching data for $5 \times 5 \mu \mathrm{m}^{2}$ capacitors by the NLS model. 
Using the extrapolation values of $v_{\infty}$ and $R_{\infty}$ from the plot in Fig. 4(b), we obtain that for $E=200 \mathrm{kV} / \mathrm{cm}$ the nucleation time becomes a rate-limiting parameter for capacitors smaller than $0.6 \mu \mathrm{m}^{2}$. This is in reasonable agreement with the size of capacitors used in present experiments $\left(1.5 \mu \mathrm{m}^{2}\right)$. As contribution of nucleation to the polarization volume switching in smaller capacitors is reduced (they switch mainly via the domain wall motion), in the high-field range the smaller capacitors will switch faster than the larger capacitors. In the low-field range, on the contrary, domain wall speed will limit the switching rate. For this reason, larger capacitors characterized by considerable contribution of nucleation mechanism to polarization reversal will be switching faster.

The scaling effect on polarization reversal mechanism is further manifested by different time dependence of switching behavior in smaller and larger capacitors. The switching behavior of $3 \times 3 \mu \mathrm{m}^{2}$ and $5 \times 5 \mu \mathrm{m}^{2}$ capacitors can be approximated by the NLS model [inset in Fig. 4(b)]: $S(t)=1-\sum F\left(\log t_{0}\right) \exp \left[-\left(t / t_{0}\right)^{n}\right]$, where $n$ is domain dimensionality equal to 2 and $F\left(\log t_{0}\right)$ is a Lorentzian distribution function of the characteristic switching time $t_{0}$ proposed by Jo et al. [24]. In contrast, the switching kinetics in smaller capacitors is close to the logarithmic time dependence and cannot be fitted by the NLS model. In the case of wall motion being the main mechanism of polarization reversal, the logarithmic time dependence can be a result of deceleration of wall propagation due to interaction of the moving domain wall with transverse acoustic phonons [11]. Indeed, it has been found that in $1 \times 1.5 \mu \mathrm{m}^{2}$ capacitors at $E=100 \mathrm{kV} / \mathrm{cm}$ the wall velocity drops from $13 \mathrm{~m} \mathrm{~s}^{-1}$ at the beginning of switching to less than $1 \mathrm{~m} \mathrm{~s}^{-1}$ at the end of switching.

In summary, inhomogeneous domain nucleation due to the defect structure of the film-electrode interface results in domain kinetics reproducible from cycle to cycle and allows application of the step-by-step switching approach to study fast polarization reversal processes. PFM visualization of domain switching kinetics makes possible for the first time direct assessment of relative contribution of nucleation and domain wall motion into polarization reversal and investigation of the scaling effect in ferroelectric capacitors. Direct measurements of the field dependent wall velocity and nucleation rate provide quantitative explanation for the higher switching speed in smaller capacitors in the high-field range. The developed approach can be applied to investigation of fast kinetic processes in multiferroic materials by means of scanning probe microscopy.

The authors acknowledge financial support of the National Science Foundation (Grant No. DMR02-35632) and thank Dr. B.J. Rodriguez for his help in PFM measurements. *alexei_gruverman@unl.edu

[1] K. R. Udayakumar, T.S. Moise, and S. R. Summerfelt et al., Jpn. J. Appl. Phys. 46, 2180 (2007).

[2] T. K. Song, S. Aggarwal, Y. Gallais, B. Nagaraj, R. Ramesh, and J. Evans, Appl. Phys. Lett. 73, 3366 (1998).

[3] S. Li, J. A. Eastman, Z. Li, C. M. Foster, R. E. Newnham, and L. E. Cross, Phys. Lett. A 212, 341 (1996).

[4] Y. Ishibashi and Y. Takagi, J. Phys. Soc. Jpn. 31, 506 (1971).

[5] M. Avrami, J. Chem. Phys. 7, 1103 (1939).

[6] A. N. Kolmogorov, Izv. Akad. Nauk USSR, Ser. Math. 3, 355 (1937).

[7] N. W. Dalton, J. T. Jacobs, and B. D. Silverman, Phys. Rev. 133, A1034 (1964).

[8] X.F. Du and I. W. Chen, Appl. Phys. Lett. 72, 1923 (1998).

[9] A. Tagantsev et al., Phys. Rev. B 66, 214109 (2002).

[10] D. J. Jung, M. Dawber, A. Ruediger, J. F. Scott, H. H. Kim, and K. Kim, Appl. Phys. Lett. 81, 2436 (2002).

[11] M. Dawber, D. J. Jung, and J. F. Scott, Appl. Phys. Lett. 82, 436 (2003).

[12] J. Li, B. Nagaraj, H. Liang, W. Cao, Chi. H. Lee, and R. Ramesh, Appl. Phys. Lett. 84, 1174 (2004).

[13] A. Grigoriev, D.-H. Do, D. M. Kim, C.-B. Eom, B. Adams, E. Dufresne, and P. G. Evans, Phys. Rev. Lett. 96, 187601 (2006).

[14] S. Tiedke, T. Schmitz, K. Prume, A. Roelofs, T. Schneller, U. Kail, R. Waser, C. Ganpule, V. Nagarajan, A. Stanishefsky, and R. Ramesh, Appl. Phys. Lett. 79, 3678 (2001).

[15] S. Prasertchoung, V. Nagarajan, Z. Ma, R. Ramesh, J. S. Cross, and M. Tsukada, Appl. Phys. Lett. 84, 3130 (2004).

[16] V. Shur, E. Rumyantsev, and S. Makarov, J. Appl. Phys. 84, 445 (1998).

[17] Y. G. Wang, W. L. Zhong, and P. L. Zhang, Phys. Rev. B 51, 17235 (1995).

[18] C. Dehoff, B. J. Rodriguez, A. I. Kingon, R. J. Nemanich, A. Gruverman, and J.S. Cross, Rev. Sci. Instrum. 76, 023708 (2005).

[19] S. Jesse, A. P. Baddorf, and S. V. Kalinin, Appl. Phys. Lett. 88, 062908 (2006).

[20] J. F. Scott et al., J. Appl. Phys. 64, 787 (1988).

[21] T. Hase and T. Shiosaki, Jpn. J. Appl. Phys. 30, 2159 (1991).

[22] Note that $3 \times 3 \mu \mathrm{m}^{2}$ and $5 \times 5 \mu \mathrm{m}^{2}$ capacitors have very close values of activation fields and switching times. Consequently, we ruled out the RC time constant as a possible reason for the different switching behavior of $1 \times$ $1.5 \mu \mathrm{m}^{2}$ capacitors.

[23] J. F. Scott et al., Proceedings of the 6th IEEE International Symposium on Applications of Ferroelectrics (IEEE, New York, 1986), p. 569; H.L. Stadler and P.J. Zachmanidis, J. Appl. Phys. 34, 3255 (1963).

[24] J. Y. Jo, H. S. Han, J.-G. Yoon, T. K. Song, S.-H. Kim, and T. W. Noh, Phys. Rev. Lett. 99, 267602 (2007). 